OPEN ACCESS

Edited by:

Scott Edward Counts, Michigan State University,

United States

Reviewed by:

Omar M. Al-Janabi,

University of Kentucky HealthCare,

United States

Cheryl R. McCreary,

University of Calgary, Canada

*Correspondence:

Sébastien Celle

sebastien.celle@univ-st-etienne.fr

Specialty section:

This article was submitted to

Neurodegeneration,

a section of the journal

Frontiers in Neuroscience

Received: 26 July 2021

Accepted: 22 November 2021

Published: 13 January 2022

Citation:

Celle S, Boutet C, Annweiler C,

Ceresetti R, Pichot $V$, Barthélémy J-C and Roche F (2022) Leukoaraiosis and Gray Matter Volume Alteration in Older Adults: The PROOF Study.

Front. Neurosci. 15:747569. doi: 10.3389/fnins.2021.747569

\section{Leukoaraiosis and Gray Matter Volume Alteration in Older Adults: The PROOF Study}

\author{
Sébastien Celle ${ }^{1,2 *}$, Claire Boutet ${ }^{3,4}$, Cédric Annweiler ${ }^{5,6}$, Romain Ceresetti ${ }^{1,2}$, \\ Vincent Pichot ${ }^{1,2}$, Jean-Claude Barthélémy ${ }^{1,2}$ and Frédéric Roche ${ }^{1,2}$ \\ ${ }^{1}$ Clinical Physiology, Visas Center, University Hospital, Saint-Etienne, France, ${ }^{2}$ INSERM, U1059, SAINBIOSE, DVH, \\ Saint-Étienne, France, ${ }^{3}$ Department of Radiology, University Hospital, Saint Etienne, France, ${ }^{4}$ EA7423 TAPE, UJM, \\ Saint-Étienne, France, ${ }^{5}$ Department of Geriatric Medicine and Memory Clinic, Research Center on Autonomy and Longevity, \\ University Hospital, Angers, France, ${ }^{6}$ UPRES EA4638, University of Angers, Angers, France
}

Background and Purpose: Leukoaraiosis, also called white matter hyperintensities $(\mathrm{WMH})$, is frequently encountered in the brain of older adults. During aging, gray matter structure is also highly affected. WMH or gray matter defects are commonly associated with a higher prevalence of mild cognitive impairment. However, little is known about the relationship between $\mathrm{WMH}$ and gray matter. Our aim was thus to explore the relationship between leukoaraiosis severity and gray matter volume in a cohort of healthy older adults.

Methods: Leukoaraiosis was rated in participants from the PROOF cohort using the Fazekas scale. Voxel-based morphometry was performed on brain scans to examine the potential link between $\mathrm{WMH}$ and changes of local brain volume. A neuropsychological evaluation including attentional, executive, and memory tests was also performed to explore cognition.

Results: Out of 315 75-year-old subjects, 228 had punctuate foci of leukoaraiosis and 62 had begun the confluence of foci. Leukoaraiosis was associated with a decrease of gray matter in the middle temporal gyrus, in the right medial frontal gyrus, and in the left parahippocampal gyrus. It was also associated with decreased performances in memory recall, executive functioning, and depression.

Conclusion: In a population of healthy older adults, leukoaraiosis was associated with gray matter defects and reduced cognitive performance. Controlling vascular risk factors and detecting early cerebrovascular disease may prevent, at least in part, dementia onset and progression.

Keywords: leukoaraiosis, aging, voxel based morphometry, cognition, vascular risk factors

\section{INTRODUCTION}

Leukoaraiosis is a rarefaction of white matter found on magnetic resonance imaging (MRI) as white matter hyperintensities (WMH) (Hachinski et al., 1987). WMH are commonly observed on brain MRIs in older adults, thus leading some authors to speak of age-related white matter changes.

Although they are considered as silent manifestations of aging, WMH do not only correlate with age but they are also associated with numerous cardiovascular risk factors including hypertension, diabetes mellitus (Scharf et al., 2019; Grosu et al., 2021), and obstructive sleep apnea 
(Ho et al., 2018). We already reported that, in older untreated prehypertensive patients, increased ambulatory blood pressure (BP) was significantly associated with higher occurrence of leukoaraiosis (Avet et al., 2014), an alteration found with a relative low mean load value of 24 -h systolic BP. Such frailty of the peripheral vascular cerebral tree in older adults challenges the general practitioners to apply a more important use of antihypertensive medication in older adults, optimal in fact, and consider the other risk factors of obstructive sleep apnea and diabetes as well. It is noticeable that these three conditions share hypertension as a common risk factor.

Furthermore, leukoaraiosis appears to be deleterious for cognitive functions, resulting in lower hippocampal volume (Triantafyllou et al., 2020) and/or higher prevalence of mild cognitive impairment, Alzheimer's disease, and dementia (Cai et al., 2015; Alber et al., 2019). The pathophysiological consequences of such alterations in deep brain white matter are still debated. It is proposed that axonal alterations linked to microvascular periventricular occlusion may in turn affect the integrity of neuronal function, with acceleration of cell death in several critical cortical areas. In 2018, Al-Janabi et al. (2018) observed that WMH were one of the major factors explaining global cerebral atrophy. Furthermore, Lambert et al. $(2015,2016)$ observed significant gray matter (GM) changes linked to WMH. According to Raji et al. (2012), the increase in white matter lesions is related to a decrease in GM volume as well as a decrease in cognition. To our knowledge, the link between $\mathrm{WMH}$, a full exhaustive exploration of cognitive decline, and GM decrease has never been studied in the healthy elderly population.

Hence, our aim in the present study was to refine this threeparty relationship between leukoaraiosis severity and GM volume decrease in a cohort representative of the general population aged 75 years and over.

\section{MATERIALS AND METHODS}

\section{Population}

The PROgnostic indicator OF cardiovascular and cerebrovascular events (PROOF) study consists of 1,011 healthy older adults homogeneous in age, 65 years, when recruited for inclusion in the city of Saint-Étienne, France (Barthélémy et al., 2007). At inclusion, in 2001, all participants were at low risk of cardiac or cerebrovascular events (2001 timepoint).

In 2009-2011, participants were invited to pass the fourth examination of the study comprising ambulatory BP monitoring and MRI of the brain. More than a half of the 1,011 subjects agreed (2010 time point).

The PROOF study was approved by an Ethics Review Board (CCPRB Rhône-Alpes Loire), and all participants signed a written informed consent for all clinical research procedures.

\section{Ambulatory Blood Pressure Monitoring}

Ambulatory blood pressure was monitored on $24 \mathrm{~h}$ using a noninvasive auscultatory method (Diasys Integra, Novacor, RueilMalmaison, France) at both time points (Avet et al., 2014).
Automatic measurements began in the early morning on a weekday and were taken from the non-dominant arm every 15 min during daytime and every 30 min during the night. During the recording, participants were required not to change their habits, including daily activities and sleeping habits.

Clinical systolic and diastolic blood pressures (SBP and DBP, respectively) were also measured and defined as the mean of two consecutive BP measurements obtained with a mercury sphygmomanometer after the subject had been lying down for $15 \mathrm{~min}$.

Average values of 24-h SBP and DBP, and awake and sleep SBP and DBP, were calculated from ambulatory blood pressure monitoring (ABPM). Systolic and diastolic Dip were calculated according to Dip = (awake BP - sleep BP/awake BP $)$; pulse pressure (PP) was defined as PP $=24$-h SBP $-24-\mathrm{h}$ DBP; and mean arterial BP (MAP) was obtained from MAP $=(24-\mathrm{h}$ $\mathrm{SBP}+[2 \times 24-\mathrm{h} \mathrm{DBP}]) / 3)$.

\section{Brain Magnetic Resonance Imaging}

MRI was performed at a second time point with a $1.5-\mathrm{T}$ scanner (Magnetom Avento, Siemens Healthcare, Erlangen, Germany), which had a 12-channel head coil. The acquisition protocol included a 3D T1 magnetization prepared rapid gradient echo (MP-RAGE) with a resolution of $1 \times 1 \times 1 \mathrm{~mm}^{3}$, repetition time (TR) of 2,060 ms, echo time (TE) of $3.23 \mathrm{~ms}$, inversion time (TI) of $1,100 \mathrm{~ms}$, and acquisition matrix of $256 \times 256$ and included 176 slices. The protocol also included a T2-weighted, 2D turbo spin echo with a TR of $6,270 \mathrm{~ms}$, TE of $109 \mathrm{~ms}$, acquisition matrix of $448 \times 360$, and 24 slices which were $5 \mathrm{~mm}$ thick. A 2D fluidattenuated inversion recovery (FLAIR) was also acquired with a TR of 5,000 ms, TE of $350 \mathrm{~ms}$, TI of $1,800 \mathrm{~ms}$, acquisition matrix of $256 \times 192$, and 52 3-mm-thick slices.

Visual examination of the MRIs lead to the exclusion of scans considered as structurally invalid for a voxel-based morphometry (VBM) study (meningiomas, major lacunas, cysts, sequels of stroke).

\section{Assessment of White Matter Lesions}

The degree of white matter lesion severity was rated using the semiquantitative visual age-related white matter changes scale (ARWMC) devised by Fazekas et al. (2002) applied to the T2-weighted FLAIR images. ARWMC was graded on a four-point scale of increasing severity: 0 , normal; 1 , punctuate foci; 2, beginning confluence of foci; and 3, large confluent areas. The reliability of the Fazekas scale is high with an intraoperator correlation coefficient of 0.85 (Leaper et al., 2001), and MR images were scored by a single neuroradiologist observer (CB) who was blinded to the participants' clinical information, including age, gender, prior imaging findings, and cardiovascular disease risk factors.

\section{Cognitive Evaluation}

Cognitive evaluation was performed using a battery of psychometric tests (Saint Martin et al., 2012). Global intellectual performance was evaluated using the Mini Mental State Examination. Cognitive complaint was investigated with a visual analog scale. We then explored more precisely three cognitive 


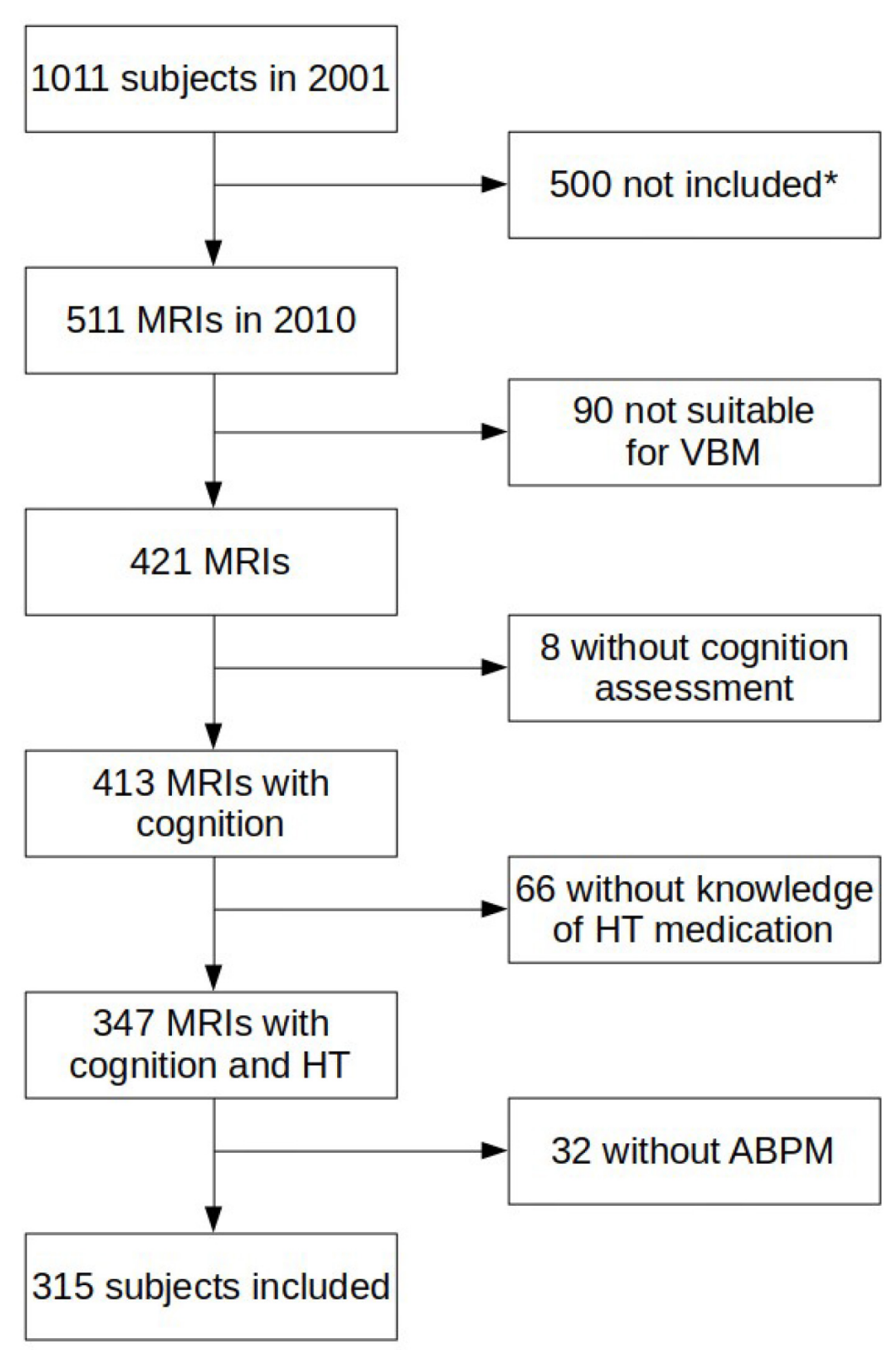

FIGURE 1 | Detailed description of the inclusion/exclusion. MRI: magnetic resonance imaging, HT: hypertension, ABPM: ambulatory blood pressure monitoring. *Missing subjects were majorly dead but were also lost from view or withdrew from the study. Others were not eligible for MRI (claustrophobia, pacemaker) or refused MRI.

domains: memory, attention and information processing, and executive functioning.

Memory was assessed using the Benton Visual Retention Test (Form C) and the Free and Cued Selective Reminding Test (FCSRT) from Grober \& Buschke. Attention and information processing were assessed using the Trail-Making Test Part A (TMTA), the Stroop Color-Word Test, and the coding subtest of the Wechsler Adult Intelligence Scale III (WAIS III). Executive functioning was tested using the Trail-Making Test Part B (TMTB), the Stroop Color-Word Test, an alphabetic fluency test using the letter $\mathrm{P}$, a category fluency test using animal names, and the similarities subtest of the WAIS III.

We also assessed anxiety and depression using the Pichot questionnaire and the French version of the Goldberg scale, respectively. Cognitive function was assessed using the short form of the MacNair scale.

\section{Voxel-Based Morphometry}

MRI images were processed according to classical SPM12 protocol using default settings. All images were first segmented using SPM12 segmentation, formerly known as NewSegment 
method. Gray matter, white matter, and cerebrospinal partitions were visually checked for mis-segmentation. Preregistered segmented white and gray matter images were registered using the DARTEL algorithm after the creation of a template based on all scans. Registered segmented images were normalized to the Montreal Neurological Institute (MNI) space and were finally smoothed with an $8^{*} 8^{*} 8-\mathrm{mm}^{3}$ kernel. Mis-registration was visually checked, and a CAT12 check sample homogeneity script was used to focus on the most different images. No subject was excluded after the visual inspections.

\section{Statistical Analysis}

For VBM analysis, we performed a multiple linear regression with SPM12: leukoaraiosis was the covariate of interest whereas sex, presence of antihypertensive medications, sociocultural level, and total intracranial volume were introduced in the model as covariates. The statistical threshold was set at $p<0.05$ at the family-wise error (FWE)-corrected voxel level with an extent threshold of 250 voxels to have the most significant clusters $(p<0.001$ at cluster level).

One-way ANOVA was used to explore the differences of cognitive tests between each level of leukoaraiosis. The Scheffe post hoc test was used to identify which of the three groups were statistically different.

\section{RESULTS}

\section{Characteristics of the Population}

From the 1,011 subjects included in 2001, five hundred did not have an MRI recording and 90 MRIs were considered as invalid. Eight more subjects were excluded because they did not have the cognition assessment. We had no information about medications in 66 more subjects. Furthermore, 32 refused the ABP monitoring or had invalid data. Three hundred and fifteen participants were finally included in the present analysis (Figure 1).

At the time of the MRI recording, participants were $75.3 \pm 0.8$ years old and slightly overweight with a BMI of $25.1 \pm 3.6$. Detailed characteristics of the population are given in Table 1.

\section{Leukoaraiosis}

Out of the 315 subjects, 25 were rated as normal on the ARWMRC, 228 were rated as having punctuate foci, and 62 were rated as presenting beginning confluence of foci. None had large confluent areas.

\section{Cognition}

Leukoaraiosis was mainly associated with differences in memory recall (3-word MMSE recall, total recall, and delayed free recall of the FCSRT) and in executive functioning (TMT-B, Code of the WAIS III) and also associated with depression (Table 2).

Post hoc analyses revealed that cognition was different between level 2 of the leukoaraiosis scale (beginning confluence) and level 1 (punctuate foci) but not between normal WM and level 1.
TABLE 1 | Characteristics of the population.

\begin{tabular}{|c|c|c|}
\hline & Mean \pm SD & [Min-Max] \\
\hline Age (years) & $75.3 \pm 0.8$ & {$[72.3-77.8]$} \\
\hline BMI $\left(\mathrm{kg} / \mathrm{m}^{2}\right)$ & $25.1 \pm 3.6$ & {$[15.1-35.8]$} \\
\hline Waist circumference (cm) & $86.9 \pm 10.8$ & {$[62-117]$} \\
\hline Fasting blood glucose (g/L) & $1.0 \pm 0.2$ & {$[0.7-2.4]$} \\
\hline Total cholesterol (g/L) & $2.3 \pm 0.4$ & [1.2-3.5] \\
\hline HDL cholesterol (g/L) & $0.7 \pm 0.8$ & {$[0.3-1.3]$} \\
\hline LDL cholesterol (g/L) & $1.4 \pm 0.3$ & {$[0.4-2.4]$} \\
\hline LDL/HDL cholesterol & $2.1 \pm 0.7$ & {$[0.3-4.2]$} \\
\hline Triglycerides (g/L) & $1.1 \pm 0.5$ & {$[0.4-4.0]$} \\
\hline SBP $(\mathrm{mmHg})$ & $133.2 \pm 19.4$ & {$[76-189]$} \\
\hline $\mathrm{DBP}(\mathrm{mmHg})$ & $85.6 \pm 15.2$ & {$[44-149]$} \\
\hline 24-h SBP (mmHg) & $117.2 \pm 13.0$ & {$[79-159]$} \\
\hline 24-h DBP (mmHg) & $71.9 \pm 7.4$ & {$[51-98]$} \\
\hline Awake SBP (mmHg) & $122.0 \pm 13.6$ & {$[84-162]$} \\
\hline Awake DBP (mmHg) & $75.2 \pm 8.3$ & {$[53-117]$} \\
\hline Sleep SBP (mmHg) & $106.0 \pm 13.9$ & {$[69-161]$} \\
\hline Sleep DBP (mmHg) & $64.7 \pm 7.9$ & {$[45-87]$} \\
\hline Systolic dip (mmHg) & $0.1 \pm 0.1$ & {$[-0.2-+0.3]$} \\
\hline Diastolic dip (mmHg) & $0.1 \pm 0.1$ & {$[-0.1-+0.5]$} \\
\hline $\mathrm{PP}(\mathrm{mmHg})$ & $45.3 \pm 9.6$ & [21-78] \\
\hline MAP (mmHg) & $87.0 \pm 8.5$ & {$[63.0-117.7]$} \\
\hline
\end{tabular}

Age, BMI, waist circumference, SBP, and DBP were available for the 315 subjects. Biological data were available for 288 subjects except $L D L, H D L$, and $L D L / H D L$ cholesterol (287 subjects). ABPM data were available for 309 subjects except for systolic and diastolic DIP (307 subjects).

$B M I$, body mass index; HDL, high-density lipoprotein; $L D L$, low-density lipoprotein; SBP, systolic blood pressure; DBP, diastolic blood pressure; PP, pulse pressure; $M A P$, mean arterial pressure; $A B P M$, ambulatory blood pressure monitoring. Data are presented as mean \pm standard deviation.

\section{Voxel-Based Morphometry}

Leukoaraiosis severity at time point 4 was inversely correlated with gray matter volume in the right middle temporal gyrus (Brodmann area 21), in the right medial frontal gyrus (Brodmann area 11), and in the left parahippocampal gyrus (Table 3 and Figure 2). Relationships were statistically significant at the cluster level as well as in the corrected voxel level.

We did not observe any gray matter increase associated with an increase in leukoaraiosis.

\section{DISCUSSION}

The present study confirmed that leukoaraiosis in older adults is associated with a decrease in GM volume in several cortical areas including the right middle temporal gyrus, the right medial frontal gyrus, and the left parahippocampal gyrus. Such decreases remained highly significant after usual corrections for confounders. Moreover, we noticed that higher leukoaraiosis was associated with a decrease in cognitive functions, mainly in memory (total recall) and executive functions (Code of the WAIS III, TMTB).

In 2001, the Leukoaraiosis And DISability (LADIS) study was built to assess the role of white matter changes and their progression on the onset of disability in older adults 
TABLE 2 | Relationship between cognitive tests and leukoaraiosis level using one-way ANOVA.

\begin{tabular}{|c|c|c|c|c|}
\hline Leukoaraiosis level & 0 & 1 & 2 & $p$-value \\
\hline Visual analog scale & $2.5 \pm 1.6$ & $2.8 \pm 1.7$ & $3.0 \pm 1.6$ & ns \\
\hline \multicolumn{5}{|l|}{ MMSE } \\
\hline Total score & $28.4 \pm 2.2$ & $28.5 \pm 1.6$ & $27.8 \pm 2.0$ & $<0.05$ \\
\hline 3-word recall & $2.5 \pm 0.8$ & $2.7 \pm 2.6$ & $2.5 \pm 0.8$ & ns \\
\hline \multicolumn{5}{|l|}{ FCSRT } \\
\hline Immediate recall & $15.4 \pm 0.9$ & $15.4 \pm 1.0$ & $15.1 \pm 1.2$ & ns \\
\hline Total recall & $46.6 \pm 1.8$ & $46.2 \pm 3.0$ & $44.7 \pm 6.6$ & $<0.05$ \\
\hline Delayed recall & $15.8 \pm 0.5$ & $15.7 \pm 0.7$ & $15.2 \pm 2.3$ & $<0.01$ \\
\hline Total free recall & $29.2 \pm 6.6$ & $29.4 \pm 5.8$ & $27.7 \pm 7.1$ & ns \\
\hline Delayed free recall & $11.7 \pm 2.4$ & $11.7 \pm 2.3$ & $11.1 \pm 3.1$ & ns \\
\hline Delayed cued recall & $4.0 \pm 2.4$ & $4.1 \pm 2.1$ & $4.2 \pm 2.4$ & ns \\
\hline Intrusions & $0.6 \pm 1.0$ & $0.7 \pm 1.4$ & $1.3 \pm 2$ & $<0.05$ \\
\hline Recognition & $16.0 \pm 0.2$ & $16.0 \pm 0.2$ & $15.8 \pm 0.7$ & $<0.001$ \\
\hline Semantically related intrusions & 0 & $0.0 \pm 0.3$ & $0.1 \pm 0.9$ & ns \\
\hline Unrelated intrusions & 0 & $0.0 \pm 0.1$ & $0.0 \pm 0.2$ & ns \\
\hline Benton test & $13.0 \pm 1.3$ & $12.6 \pm 1.6$ & $12.5 \pm 1.6$ & ns \\
\hline \multicolumn{5}{|l|}{ WAIS-III code test } \\
\hline Score & $50.9 \pm 9.6$ & $53.6 \pm 12.6$ & $49.0 \pm 12.8$ & $<0.05$ \\
\hline Standardized score & $11.0 \pm 2.1$ & $11.6 \pm 2.6$ & $10.6 \pm 2.8$ & $<0.05$ \\
\hline Copy score & $110.0 \pm 23.4$ & $114.2 \pm 20.5$ & $110.2 \pm 23.9$ & ns \\
\hline \multicolumn{5}{|l|}{ Trail making test } \\
\hline TMT-A (s) & $49.7 \pm 15.1$ & $46.9 \pm 15.4$ & $49.9 \pm 16.6$ & ns \\
\hline TMT-A Errors & $0.0 \pm 0.2$ & $0.1 \pm 0.8$ & $0.1 \pm 0.2$ & ns \\
\hline TMT-B (s) & $102.3 \pm 33.2$ & $98.7 \pm 35.8$ & $114.9 \pm 58.7$ & $7<0.05$ \\
\hline TMT-B Errors & $0.2 \pm 0.4$ & $0.5 \pm 1.0$ & $0.7 \pm 1.2$ & ns \\
\hline TMT-B-TMT-A (s) & $52.6 \pm 25.6$ & $51.8 \pm 30.3$ & $65.0 \pm 49.2$ & $<0.05$ \\
\hline \multicolumn{5}{|l|}{ Stroop } \\
\hline Word score & $89.4 \pm 16.9$ & $94.5 \pm 13.0$ & $93.0 \pm 11.7$ & ns \\
\hline Color score & $65.9 \pm 11.4$ & $66.7 \pm 10.7$ & $64.6 \pm 8.9$ & ns \\
\hline Color-word score & $32.3 \pm 8.2$ & $32.4 \pm 7.9$ & $31.3 \pm 9.1$ & ns \\
\hline Stroop Interference & $3.2 \pm 6.6$ & $2.1 \pm 6.7$ & $2.1 \pm 8.0$ & ns \\
\hline Standardized word score & $103.4 \pm 16.9$ & $108.6 \pm 13.0$ & $107.1 \pm 11.7$ & ns \\
\hline Word z-score & $-0.1 \pm 0.4$ & $-0.1 \pm 0.7$ & $-0.1 \pm 0.5$ & ns \\
\hline Standardized color score & $75.9 \pm 11.4$ & $77.6 \pm 10.7$ & $75.6 \pm 8.9$ & ns \\
\hline Color z-score & $-0.6 \pm 0.7$ & $-0.3 \pm 0.7$ & $-0.3 \pm 0.7$ & ns \\
\hline Standardized color-word score & $47.2 \pm 8.3$ & $47.3 \pm 7.9$ & $46.3 \pm 9.0$ & ns \\
\hline Color-word z-score & $-0.2 \pm 0.6$ & $0.1 \pm 0.8$ & $-0.4 \pm 1.0$ & ns \\
\hline \multicolumn{5}{|l|}{ Verbal fluency } \\
\hline Semantic & $30.2 \pm 8.0$ & $30.4 \pm 7.3$ & $28.5 \pm 7.7$ & ns \\
\hline Semantic false answer & $0.8 \pm 0.9$ & $0.6 \pm 1.0$ & $0.5 \pm 0.8$ & ns \\
\hline Semantic Z-score & $0.1 \pm 0.9$ & $0.1 \pm 0.9$ & $-0.2 \pm 1.0$ & ns \\
\hline Phonemic & $21.0 \pm 8.3$ & $20.4 \pm 6.3$ & $19.0 \pm 6.4$ & ns \\
\hline Phonemic false answer & $0.6 \pm 0.8$ & $0.6 \pm 0.9$ & $0.4 \pm 0.8$ & ns \\
\hline Phonemic Z-score & $0.1 \pm 1.0$ & $-0.1 \pm 0.9$ & $-0.2 \pm 0.7$ & ns \\
\hline \multicolumn{5}{|l|}{ WAIS-III similarities test } \\
\hline Score & $19.5 \pm 5.4$ & $17.7 \pm 5.6$ & $17.1 \pm 5.8$ & ns \\
\hline Standardized score & $12.3 \pm 2.4$ & $11.4 \pm 2.5$ & $11.2 \pm 2.8$ & $\mathrm{~ns}$ \\
\hline Z-score & $0.8 \pm 0.8$ & $0.5 \pm 0.8$ & $0.4 \pm 0.9$ & ns \\
\hline Cognitive function & $25.6 \pm 8.7$ & $26.8 \pm 11.2$ & $29.3 \pm 13.9$ & ns \\
\hline Anxiety & $2.9 \pm 2.6$ & $3.1 \pm 2.8$ & $3.0 \pm 2.6$ & ns \\
\hline Depression & $2.0 \pm 1.7$ & $2.3 \pm 2.4$ & $3.5 \pm 3.7$ & $<0.001$ \\
\hline
\end{tabular}

MMSE, Mini-Mental State Examination; TMT, trail making test; FCRST, Free and Cued Selective Reminding Test. Data are presented as mean \pm standard deviation.
TABLE 3 | Gray matter decrease associated with an increase in leukoaraiosis score (R: right, L: left, pclust: $p$-value at the cluster level with a family-wise error (FWE) correction, pvox: $p$-value at the peak voxel with a FWE correction).

\begin{tabular}{lccccccc}
\hline Side & Brain region & $\mathbf{X}$ & $\mathbf{Y}$ & $\mathbf{Z}$ & Cluster size & pclust & pvox \\
\hline $\mathrm{R}$ & Middle temporal gyrus & 54 & -8 & -22 & 282 & 0.001 & $<0.001$ \\
$\mathrm{R}$ & Medial frontal gyrus & 3 & 33 & -14 & 337 & 0.001 & 0.002 \\
$\mathrm{~L}$ & Parahippocampal gyrus & -32 & -24 & -15 & 265 & 0.001 & 0.007 \\
\hline
\end{tabular}

(The LADIS Study Group, 2011). In 639 non-healthy but functionally autonomous 74-year-old patients, the group demonstrated that WMH were associated with worse score on MMSE (van der Flier, 2005) and worse cognitive performance (Verdelho et al., 2007). However, these subjects had various complaints and disturbances (mild cognitive impairment, motor disturbances, mood disturbances, minor cerebrovascular events, or other neurological problems). Later, the association between WMH and cognitive impairment and/or dementia was confirmed in older adults (94 years old) (Legdeur et al., 2020). A meta-analysis in more than 14,000 participants for WMH showed a link between WMH and dementia/Alzheimer's disease (AD) (Debette et al., 2019). In 2004, Verdelho et al. (2007) observed an association between WMH and Buschke total recall. We confirmed this association in a healthy 75-year-old population comprising adults without any major cognitive impairment or dementia or cognitive complaint (Capizzano, 2004). The differences observed here are subtle, but they remain statistically significant. Of note, none of the subjects had large confluent areas, as in the publication of Verdelho et al. (2007), while we still noted these significant cognitive variations.

Interestingly, Kim et al. (2020) did not observe any relationship between $\mathrm{WMH}$ and cognition on subcortical vascular mild cognitive impairment patients. However, they demonstrated a more rapid cortical thinning in frontal and temporal areas associated with a 3-year WMH progression. Cortical thinning in frontal and temporal areas associated with white matter grade was reported by Raji et al. (2012) as well as a thinning in inferior parietal and parahippocampal areas. For Dickie et al. (2016) WMH were also associated with a cortical thinning within and around the Sylvian fissure. In 2005, van der Flier (2005) from the LADIS study group showed that those with medial temporal atrophy and severe WMH had a fourfold increase in the frequency in mild cognitive deficits. In a meta-analysis by Wang et al. (2021) WMH were also associated with medial temporal lobe atrophy in post-stroke patients. Li et al. (2011) also observed frontal and temporal lobe GM decreases using VBM analysis, as well as decreases in the thalamus and parietal lobe. Several studies reported a link between WMH and hippocampal atrophy in mild cognitive impairment patients (Fiford et al., 2017; Wong et al., 2021). In our study, we also observed a GM volume decrease in similar areas (middle temporal gyrus, medial frontal gyrus) as well as in the parahippocampus. Using linear mixed effect models, van Leijsen and her team explained memory decline in older 
A

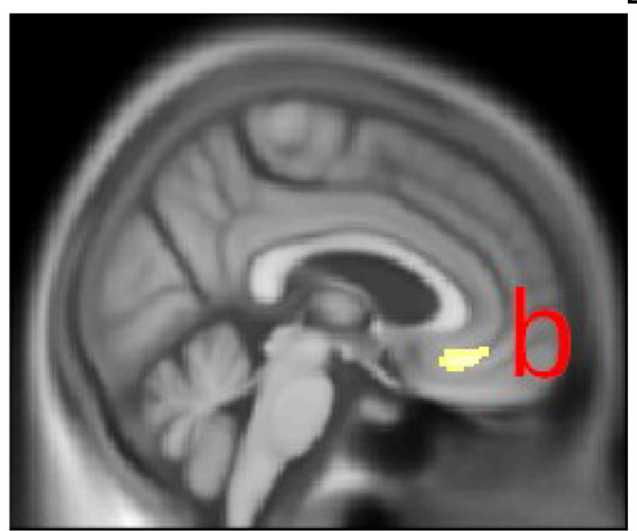

C

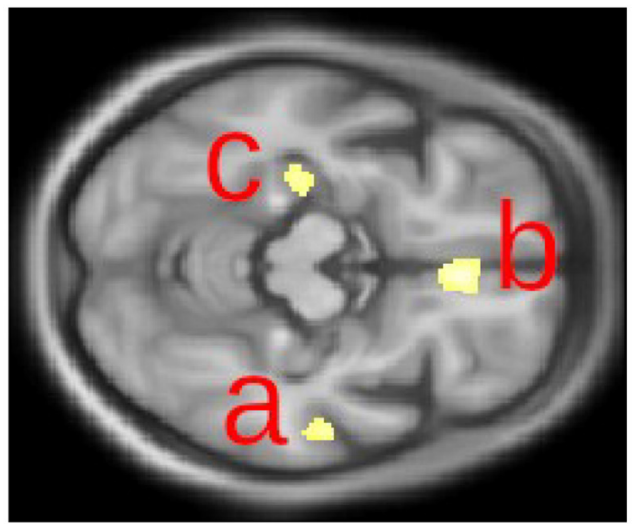

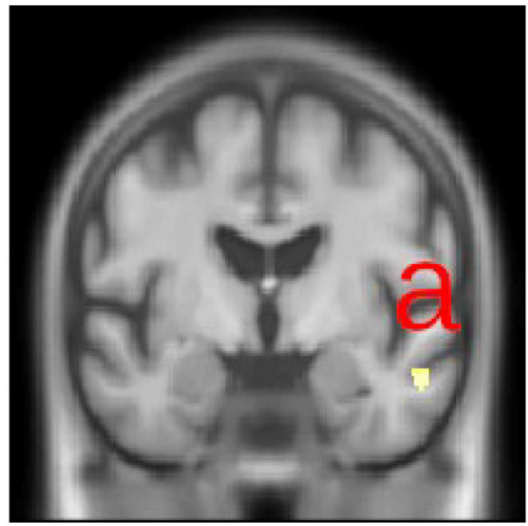

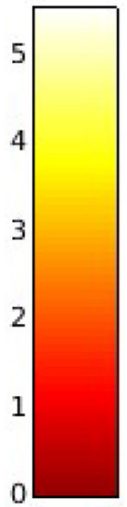

FIGURE 2 | Gray matter volume decrease in the right middle temporal gyrus (A), right medial frontal gyrus (B), and left parahippocampal gyrus (C).

adults by an interaction between WMH and hippocampus volume (van Leijsen et al., 2019). WMH and hippocampus volume were also both markers of the conversion from normal cognition to mild cognitive impairment in Framingham offsprings (Bangen et al., 2018).

During the past decades, $\mathrm{WMH}$ have been associated with the two most common forms of dementia, vascular dementia (VD) and $\mathrm{AD}$. The first form of dementia, $\mathrm{VD}$, is caused by defects in blood supply to the brain which generates lacunae and $\mathrm{WMH}$. The second form of dementia, $\mathrm{AD}$, is a multifactorial neurodegenerative disease whose main risk factors are age, genetic, head injury, and other factors such as high cholesterol or high BP. There is a link between AD and/or dementia and $\mathrm{WMH}$, even after the concomitant effect of age on both pathologies had been dismissed (Sarabia-Cobo et al., 2014; Kao et al., 2019). White matter alterations appear years before the estimated symptomatic onset of the disease in autosomal dominant AD (Lee et al., 2016; Araque Caballero et al., 2018). Rojas et al. (2018) discovered a higher prevalence of $\mathrm{WMH}$ in homozygous APOE- 44 allele carriers aged 4575 years. In non-genetic forms of $\mathrm{AD}$, this link has been less documented and $\mathrm{WMH}$ are only associated with severity of AD. Temporal areas and parahippocampus/hippocampus are the first regions affected in $\mathrm{AD}$. It has been hypothesized that the presence of high $\mathrm{WMH}$ and the decrease of gray matter in key brain areas may be viewed as prodromal signs of $\mathrm{AD}$ as suggested in the literature (Wolf et al., 2000; Vermeer et al., 2003; Prins et al., 2004). It is of importance to note that our population is highly selected: among the 1,011 healthy participants included in 2001, the 315 participants remaining in this sub-study are probably the healthiest part of the population. Thus, they have shown few WMH and they mainly do not have cognitive impairment. None of these participants suffers from dementia, and we do not yet know whether those with poor cognitive results will convert to dementia or not. Future time points of our study should answer this specific question.

WMH are associated with hypertension, dyslipidemia, or diabetes, suggesting a vascular origin of leukoaraiosis in which hypertension seems to play a major key role (Moroni et al., 2016). As a manifestation of cerebral small vessel disease, leukoaraiosis seems to develop gradually and be the "tip of the iceberg" according to de Groot et al. (2013). Conventional MRI techniques may be not sensible enough to detect the first brain disorders: Altamura et al. (2016) observed on MRIs that even in patients with normal-appearing white matter, higher regional apparent water diffusion coefficients were associated with microcirculatory impairment and altered cognitive domain. It thus seems that WMH can originate from cerebral blood flow decrease (Bernbaum et al., 2015). In a previous study, we 
observed a linear relationship between leukoaraiosis and $\mathrm{BP}$ in untreated for hypertension patients, but not in patients treated for hypertension (Avet et al., 2014), which can suggest that controlling hypertension could lower WMH burden.

However, in our study we only assessed WMH using the Fazekas scale, one among multiple ways to define WMH (Frey et al., 2019). Measurements of WMH volumes or local variations of WMH may bring sharper results. Moreover, we were unable to evaluate the status of our population regarding microbleeds.

As a conclusion, WMH are associated with cognitive impairment and brain GM defects in healthy older adults. As WMH find their origin in cerebrovascular impairment, this result strengthens the assumption by Sudo et al. (2015) that controlling vascular risk factors and detecting early cerebrovascular diseases may prevent, at least in part, dementia onset and progression. Further interventional studies implying the strict control of vascular risk factors and their impact on the brain and dementia should confirm this conclusion.

\section{REFERENCES}

Alber, J., Alladi, S., Bae, H. J., Barton, D. A., Beckett, L. A., Bell, J. M., et al. (2019). White matter hyperintensities in Vascular Contributions to Cognitive Impairment and Dementia (VCID): knowledge gaps and opportunities. Alzheimers Dement. 5, 107-117. doi: 10.1016/j.trci.2019. 02.001

Al-Janabi, O. M., Panuganti, P., Abner, E. L., Bahrani, A. A., Murphy, R., Bardach, S. H., et al. (2018). Global cerebral atrophy detected by routine imaging: relationship with age, hippocampal atrophy, and white matter hyperintensities. J. Neuroimaging 28, 301-306. doi: 10.1111/jon.12494

Altamura, C., Scrascia, F., Quattrocchi, C. C., Errante, Y., Gangemi, E., Curcio, G., et al. (2016). Regional MRI diffusion, white-matter hyperintensities, and cognitive function in alzheimer's disease and vascular dementia. J. Clin. Neurol. 12:201. doi: 10.3988/jcn.2016.12.2.201

Araque Caballero, M. Á, Suárez-Calvet, M., Duering, M., Franzmeier, N., Benzinger, T., Fagan, A. M., et al. (2018). White matter diffusion alterations precede symptom onset in autosomal dominant alzheimer's disease. Brain 141, 3065-3080. doi: 10.1093/brain/awy229

Avet, J., Pichot, V., Barthélémy, J. C., Laurent, B., Garcin, A., Roche, F., et al. (2014). Leukoaraiosis and ambulatory blood pressure load in a healthy elderly cohort study: the PROOF study. Int. J. Cardiol. 172, 59-63. doi: 10.1016/j.ijcard.2013. 11.052

Bangen, K. J., Preis, S. R., Delano-Wood, L., Wolf, P. A., Libon, D. J., Bondi, M. W., et al. (2018). Baseline white matter hyperintensities and hippocampal volume are associated with conversion from normal cognition to mild cognitive impairment in the framingham offspring study. Alzheimer Dis. Assoc. Disord. 32, 50-56. doi: 10.1097/WAD.0000000000000215

Barthélémy, J. C., Pichot, V., Dauphinot, V., Celle, S., Laurent, B., Garcin, A., et al. (2007). Autonomic nervous system activity and decline as prognostic indicators of cardiovascular and cerebrovascular events: the "PROOF" study. study design and population sample. associations with sleep-related breathing disorders: the "SYNAPSE" study. Neuroepidemiology 29, 18-28. doi: 10.1159/0001 08914

Bernbaum, M., Menon, B. K., Fick, G., Smith, E. E., Goyal, M., Frayne, R., et al. (2015). Reduced blood flow in normal white matter predicts development of leukoaraiosis. J. Cereb. Blood Flow Metab. 35, 1610-1615. doi: 10.1038/jcbfm. 2015.92

Cai, Z., Wang, C., He, W., Tu, H., Tang, Z., Xiao, M., et al. (2015). Cerebral small vessel disease and alzheimers disease. Clin. Interv. Aging 10, 1695-1704. doi: 10.2147/CIA.S90871

\section{DATA AVAILABILITY STATEMENT}

The generated datasets may be available after a motivated request to the research team. Requests to access the datasets should be directed to FR.

\section{ETHICS STATEMENT}

The studies involving human participants were reviewed and approved by the CCPRB Rhône-Alpes Loire. The patients/participants provided their written informed consent to participate in this study.

\section{AUTHOR CONTRIBUTIONS}

SC and CB contributed to the analysis and interpretation of data. $\mathrm{J}-\mathrm{CB}$ and FR contributed to the conception and design of the study. SC drafted the manuscript. All authors critically revised the draft.

Capizzano, A. A. (2004). White matter hyperintensities are significantly associated with cortical atrophy in alzheimer's disease. J. Neurol. Neurosurg. Psychiatry 75, 822-827. doi: 10.1136/jnnp.2003.019273

de Groot, M., Verhaaren, B. F., de Boer, R., Klein, S., Hofman, A., van der Lugt, A., et al. (2013). Changes in normal-appearing white matter precede development of white matter lesions. Stroke 44, 1037-1042. doi: 10.1161/STROKEAHA.112. 680223

Debette, S., Schilling, S., Duperron, M. G., Larsson, S. C., and Markus, H. S. (2019). Clinical significance of magnetic resonance imaging markers of vascular brain injury: a systematic review and meta-analysis. JAMA Neurol. 76, 81. doi: 10.1001/jamaneurol.2018.3122

Dickie, D. A., Karama, S., Ritchie, S. J., Cox, S. R., Sakka, E., Royle, N. A., et al. (2016). Progression of white matter disease and cortical thinning are not related in older community-dwelling subjects. Stroke 47, 410-416. doi: 10.1161/STROKEAHA.115.011229

Fazekas, F., Barkhof, F., Wahlund, L. O., Pantoni, L., Erkinjuntti, T., Scheltens, P., et al. (2002). CT and MRI rating of white matter lesions. Cerebrovasc. Dis. 13, 31-36. doi: 10.1159/000049147

Fiford, C. M., Manning, E. N., Bartlett, J. W., Cash, D. M., Malone, I. B., Ridgway, G. R., et al. (2017). White matter hyperintensities are associated with disproportionate progressive hippocampal atrophy: association of wmh with hippocampal atrophy. Hippocampus 27, 249-262. doi: 10.1002/hipo. 22690

Frey, B. M., Petersen, M., Mayer, C., Schulz, M., Cheng, B., and Thomalla, G. (2019). Characterization of white matter hyperintensities in large-scale MRIstudies. Front. Neurol. 10:238. doi: 10.3389/fneur.2019.00238

Grosu, S., Rospleszcz, S., Hartmann, F., Habes, M., Bamberg, F., Schlett, C. L., et al. (2021). Associated factors of white matter hyperintensity volume: a machinelearning approach. Sci. Rep. 11:2325. doi: 10.1038/s41598-021-81883-4

Hachinski, V. C., Potter, P., and Merskey, H. (1987). Leuko-araiosis. Arch. Neurol. 44, 21-23. doi: 10.1001/archneur.1987.00520130013009

Ho, B. L., Tseng, P. T., Lai, C. L., Wu, M. N., Tsai, M. J., Hsieh, C. F., et al. (2018). Obstructive sleep apnea and cerebral white matter change: a systematic review and meta-analysis. J. Neurol. 265, 1643-1653. doi: 10.1007/s00415-018-8895-7

Kao, Y. H., Chou, M. C., Chen, C. H., and Yang, Y. H. (2019). White matter changes in patients with alzheimer's disease and associated factors. J. Clin. Med. 8:167. doi: $10.3390 / \mathrm{jcm} 8020167$

Kim, S. J., Lee, D. K., Jang, Y. K., Jang, H., Kim, S. E., Cho, S. H., et al. (2020). The effects of longitudinal white matter hyperintensity change on cognitive decline and cortical thinning over three years. J. Clin. Med. 9:2663. doi: 10.3390/ jcm9082663 
Lambert, C., Benjamin, P., Zeestraten, E., Lawrence, A. J., Barrick, T. R., and Markus, H. S. (2016). Longitudinal patterns of leukoaraiosis and brain atrophy in symptomatic small vessel disease. Brain 139, 1136-1151. doi: 10.1093/brain/ aww009

Lambert, C., Sam Narean, J., Benjamin, P., Zeestraten, E., Barrick, T. R., and Markus, H. S. (2015). Characterising the grey matter correlates of leukoaraiosis in cerebral small vessel disease. NeuroImage Clin. 9, 194-205. doi: 10.1016/j. nicl.2015.07.002

Leaper, S. A., Murray, A. D., Lemmon, H. A., Staff, R. T., Deary, I. J., Crawford, J. R., et al. (2001). Neuropsychologic correlates of brain white matter lesions depicted on MR images: 1921 aberdeen birth cohort. Radiology 221, 51-55. doi: 10.1148/radiol.2211010086

Lee, S., Viqar, F., Zimmerman, M. E., Narkhede, A., Tosto, G., Benzinger, T. L., et al. (2016). White matter hyperintensities are a core feature of alzheimer's disease: evidence from the dominantly inherited alzheimer network: white matter hyperintensities in familial AD. Ann. Neurol. 79, 929-939. doi: 10.1002/ ana. 24647

Legdeur, N., Badissi, M., Yaqub, M., Beker, N., Sudre, C. H., Ten Kate, M., et al. (2020). What determines cognitive functioning in the oldest-old? The EMIFAD 90+ study. J. Gerontol. B Psychol. Sci. Soc. Sci. 76, 1499-1511. doi: 10.1093/ geronb/gbaa152

Li, C., Du, H., Zheng, J., and Wang, J. (2011). A voxel-based morphometric analysis of cerebral gray matter in subcortical ischemic vascular dementia patients and normal aged controls. Int. J. Med. Sci. 8, 482-486. doi: 10.7150/ijms.8.482

Moroni, F., Ammirati, E., Magnoni, M., D’Ascenzo, F., Anselmino, M., Anzalone, N., et al. (2016). Carotid atherosclerosis, silent ischemic brain damage and brain atrophy: a systematic review and meta-analysis. Int. J. Cardiol. 223, 681-687. doi: 10.1016/j.ijcard.2016.08.234

Prins, N. D., van Dijk, E. J., den Heijer, T., Vermeer, S. E., Koudstaal, P. J., Oudkerk, M., et al. (2004). Cerebral white matter lesions and the risk of dementia. Arch. Neurol. 61:1531. doi: 10.1001/archneur.61.10.1531

Raji, C. A., Lopez, O. L., Kuller, L. H., Carmichael, O. T., Longstreth, W. T. Jr., Gach, H. M., et al. (2012). White matter lesions and brain gray matter volume in cognitively normal elders. Neurobiol. Aging 33, 834.e7-16. doi: 10.1016/j. neurobiolaging.2011.08.010

Rojas, S., Brugulat-Serrat, A., Bargalló, N., Minguillón, C., Tucholka, A., Falcon, C., et al. (2018). Higher prevalence of cerebral white matter hyperintensities in homozygous APOE- $\varepsilon 4$ allele carriers aged 45-75: results from the ALFA study. J. Cereb. Blood Flow Metab. 38, 250-261. doi: 10.1177/0271678X17707397

Saint Martin, M., Sforza, E., Barthélémy, J. C., Thomas-Anterion, C., and Roche, F. (2012). Does subjective sleep affect cognitive function in healthy elderly subjects? Proof Cohort. Sleep Med. 13, 1146-1152. doi: 10.1016/j.sleep.2012. 06.021

Sarabia-Cobo, C. M., Pérez, V., Hermosilla, C., Nuñez, M. J., and de Lorena, P. (2014). Apathy and leukoaraiosis in mild cognitive impairment and alzheimer's disease: multicenter diagnostic criteria according to the latest studies. Dement. Geriatr. Cogn. Dis. Extra 4, 228-235. doi: 10.1159/000363227

Scharf, E. L., Graff-Radford, J., Przybelski, S. A., Lesnick, T. G., Mielke, M. M., Knopman, D. S., et al. (2019). Cardiometabolic health and longitudinal progression of white matter hyperintensity: the mayo clinic study of aging. Stroke 50, 3037-3044. doi: 10.1161/STROKEAHA.119.025822

Sudo, F. K., Alves, G. S., Tiel, C., Ericeira-Valente, L., Moreira, D. M., and Laks, J. (2015). Neuroimaging criteria and cognitive performance in vascular mild cognitive impairment: a systematic review. Dement. Neuropsychol. 9, 394-404. doi: 10.1590/1980-57642015DN94000394

The LADIS Study Group (2011). 2001-2011: a decade of the LADIS (Leukoaraiosis And DISability) study: what have we learned about white matter changes and small-vessel disease? Cerebrovasc. Dis. 32, 577-588. doi: 10.1159/0003 34498

Triantafyllou, A., Ferreira, J. P., Kobayashi, M., Micard, E., Xie, Y., KearneySchwartz, A., et al. (2020). Longer duration of hypertension and MRI microvascular brain alterations are associated with lower hippocampal volumes in older individuals with hypertension. J. Alzheimers Dis. 74, 227-235. doi: 10.3233/JAD- 190842

van der Flier, W. M. (2005). Medial Temporal lobe atrophy and white matter hyperintensities are associated with mild cognitive deficits in non-disabled elderly people: the LADIS study. J. Neurol. Neurosurg. Psychiatry 76, 1497-1500. doi: 10.1136/jnnp.2005.064998

van Leijsen, E. M. C., Tay, J., van Uden, I. W. M., Kooijmans, E. C. M., Bergkamp, M. I., van der Holst, H. M., et al. (2019). Memory decline in elderly with cerebral small vessel disease explained by temporal interactions between white matter hyperintensities and hippocampal atrophy. Hippocampus 29, 500-510. doi: 10.1002/hipo.23039

Verdelho, A., Madureira, S., Ferro, J. M., Basile, A. M., Chabriat, H., Erkinjuntti, T., et al. (2007). Differential impact of cerebral white matter changes, diabetes, hypertension and stroke on cognitive performance among non-disabled elderly. the LADIS study. J. Neurol. Neurosurg. Psychiatry 78, 1325-1330. doi: 10.1136/ jnnp.2006.110361

Vermeer, S. E., Prins, N. D., den Heijer, T., Hofman, A., Koudstaal, P. J., and Breteler, M. M. (2003). Silent brain infarcts and the risk of dementia and cognitive decline. N. Engl. J. Med. 348, 1215-1222. doi: 10.1056/NEJMoa02 2066

Wang, F., Hua, S., Zhang, Y., Yu, H., Zhang, Z., Zhu, J., et al. (2021). Association between small vessel disease markers, medial temporal lobe atrophy and cognitive impairment after stroke: a systematic review and meta-analysis. J. Stroke Cerebrovasc. Dis. 30:105460. doi: 10.1016/j.jstrokecerebrovasdis.2020. 105460

Wolf, H., Ecke, G. M., Bettin, S., Dietrich, J., and Gertz, H. J. (2000). Do white matter changes contribute to the subsequent development of dementia in patients with mild cognitive impairment? A longitudinal study. Int. J. Geriatr. Psychiatry 15, 803-812. doi: 10.1002/1099-1166(200009)15:9<803::aidgps $190<3.0 . c 0 ; 2-\mathrm{w}$

Wong, F. C. C., Yatawara, C., Low, A., Foo, H., Wong, B. Y. X., Lim, L., et al. (2021). Cerebral small vessel disease influences hippocampal subfield atrophy in mild cognitive impairment. Transl. Stroke Res. 12, 284-292. doi: 10.1007/ s12975-020-00847-4

Conflict of Interest: The authors declare that the research was conducted in the absence of any commercial or financial relationships that could be construed as a potential conflict of interest.

Publisher's Note: All claims expressed in this article are solely those of the authors and do not necessarily represent those of their affiliated organizations, or those of the publisher, the editors and the reviewers. Any product that may be evaluated in this article, or claim that may be made by its manufacturer, is not guaranteed or endorsed by the publisher.

Copyright (c) 2022 Celle, Boutet, Annweiler, Ceresetti, Pichot, Barthélémy and Roche. This is an open-access article distributed under the terms of the Creative Commons Attribution License (CC BY). The use, distribution or reproduction in other forums is permitted, provided the original author(s) and the copyright owner(s) are credited and that the original publication in this journal is cited, in accordance with accepted academic practice. No use, distribution or reproduction is permitted which does not comply with these terms. 